\title{
Article \\ A Power-Assisted Cart with the Optimal Assistance Ratio and Disturbance Observer-Based Methods for Walking Assistance Applications
}

\author{
Xianglong Wan *, Jiaxin Ma, Yichi Zhang, Takahiro Endo and Fumitoshi Matsuno \\ Department of Mechanical Engineering and Science, Graduate School of Engineering, Kyoto University, \\ Kyoto daigaku-katsura, Nishikyo-ku, Kyoto 615-8530, Japan; majiaxin4321@outlook.com (J.M.); \\ zhangyichi4321@outlook.com (Y.Z.); endo@me.kyoto-u.ac.jp (T.E.); matsuno.fumitoshi.8n@kyoto-u.ac.jp (F.M.) \\ * Correspondence: wanxianglong@ustb.edu.cn
}

check for updates

Citation: Wan, X.; Ma, J.;

Zhang, Y.; Endo, T.; Matsuno, F.

A Power-Assisted Cart with the Optimal Assistance Ratio and Disturbance Observer-Based Methods for Walking Assistance Applications. Appl. Sci. 2021, 11, 1079. https://doi. org/10.3390/app11031079

Academic Editor: Ioannis Kostavelis Received: 31 December 2020

Accepted: 21 January 2021

Published: 25 January 202

Publisher's Note: MDPI stays neutral with regard to jurisdictional claims in published maps and institutional affiliations.

Copyright: (C) 2021 by the authors Licensee MDPI, Basel, Switzerland. This article is an open access article distributed under the terms and conditions of the Creative Commons Attribution (CC BY) license (https:// creativecommons.org/licenses/by/ $4.0 /)$.
Abstract: In this paper, we propose two control methods for driving a power-assisted cart made for walking assistance for the elderly. The optimal assistance ratio (OAR) and disturbance observer-based (DOB) methods properly adjust the motor output of the cart with high operational efficiency in response to changes in the environment. Healthy subjects walked with the cart on several road surfaces under various conditions, and the experimental results indicate the high operational efficiency of the two proposed methods. Meanwhile, their drawbacks are also discussed herein. The two methods can be used separately or combined according to the application. The OAR method is more suitable for indoor use, while the DOB method is applicable for outdoor applications. Combining these two methods could overcome the mentioned drawbacks.

Keywords: power-assisted cart; road roughness; assisted walking; operational efficiency; human-robot interaction

\section{Introduction}

Nowadays, the world is facing the challenge of an aging population. Especially in Japan, people aged over 65 accounted for $26.0 \%$ of the total population as of 1 October 2014 [1]. The aging population has led to higher healthcare costs and social spending because the elderly tend to have a greater need for medical and livelihood support, which has recently attracted interest from many researchers. To help the elderly and handicapped people maintain their independence and avoid social isolation, walking assistance devices such as the lower limb exoskeleton [2], the powered wheelchair [3], and the assistive cane [4] have been developed so far. While the power wheelchair is designed for those who have difficulty walking, the assistive cane provides assistance with guidance for visually impaired people.

There is another type of walking assisting device, the power-assisted cart/walker, oriented toward healthy people. The user pushes the handle of the cart to operate it. Most of the studies on power-assisted carts are on those made for industrial use [5-7]. The cart helps the operator to carry and transport extremely heavy loads, and the development objectives for the cart have been about how to stabilize the balance, reduce lateral deviation, and so on. Moreover, some researchers have focused on a cart-type assisting device for use in the daily lives of the elderly. An active robotic walker called "JARoW" has been proposed to adjust its motion direction and velocity to the user by using IR sensors [8]. Fontanelli et al. [9] proposed a guidance mechanism for a walking robotic assistant based on the use of differential braking, while Ko et al. [10] designed a control method for a walking assistance robot for outdoor guidance that enabled movement on flat ground and up and down slopes.

The intention of the user is extremely important for power-assisted devices that is highly related to the safety and effectiveness of power assistance [11,12]. While electromyo- 
graphy signals are commonly utilized for power-assisted exoskeletons [13-15], the interaction force between the user and the device is acquired for the control of the cart/walker. Yu et al. [16] developed a personal mobility aid and health monitoring system consisting of a cane/walker robot with a force/torque sensor and a proposed admittance-based control method. Kobayashi et al. [17] proposed a control method with operational prediction. However, uncertainties such as environmental variation have not been considered and so would become limitations of these methods in practical applications.

In a complex outdoor environment, road roughness is one factor that affects the operation and thus needs to be estimated and eliminated [18-20]. As the most common control strategy of the power-assisted systems on the market, such as in electric bicycles [21,22], the actuator generates a driving force proportional to the operational force from the user, and the ratio of the driving force to the operational one is usually set to be constant. However, this kind of simple strategy has several limitations when applied to power-assisted carts, especially when walking outside. For example, the necessary operational force varies with the road condition: if the road surface is rougher, the force will be larger. Moreover, while walking on a slope, the effect of gravitation needs to be compensated for. However, the weight of the load on the cart is usually unknown in daily life. Hence, if the ratio remains unchanged, the user must put more load onto the cart to exert a larger force. Thus, improper motor output will result in excessive fatigue and discomfort, which is adverse to outdoor activities. When taking the power-assisted cart as the payload, the way of maintaining the efficiency during assisted walking is the major problem in this application.

Different from the above studies, the power-assisted cart in this paper is designed for such a scene: it can carry the belongings of the user, sustain the weight of the user, and provide enough driving force with only minimal effort needed from the user to operate it. This will make it more convenient for the elderly to go outdoors and to participate in social activities, and thus is good for health [23,24] and the avoidance of cognitive impairment such as dementia $[25,26]$.

The main objective is to ensure that the cart motor delivers proper walking assistance force under various environmental conditions, including gravity and frictional resistance variation, with high efficiency.

Inspired by the fuel efficiency of cars, we propose a performance index for the assessment of operational efficiency while walking with the power-assisted cart (payload). We designed two control methods, namely the optimal assistance ratio (OAR) and the disturbance-observer-based (DOB) methods, for a power-assisted cart named RT.1 developed by Funai Electric Co., Ltd., and RT.WORKS Co., Ltd. Both assist the user by reducing the influence of the environment. The performances of these two methods were evaluated via experimentation by comparing cases without assistance and with default assistance. Eight participants walked with the power-assisted cart at a fixed pace. The performance index for each method to evaluate the operational efficiency and walking speed during assisted walking was calculated from the obtained experimental data. Finally, the drawbacks and applicable scope of the proposed methods are discussed herein.

The OAR method is based on a simple idea. Since frictional resistance varies with the road conditions, it is assumed that there is an OAR for each type of road from the data obtained in preliminary studies. Next, a classifier is chosen to recognize the road surfaces with high accuracy. After that, high efficiency needs to hold while walking with the cart on one of these surfaces.

The usefulness of the DOB method for walking assistance has been shown in a number of studies [27-30]. The key idea is that a disturbance observer can estimate and eliminate any existing "disturbance". This method has some distinctive merits compared to the OAR one. First, there is no need to estimate the road type; second, it can deal with frictional resistance and gravitational effect together; and third, it still works when no one is operating the cart.

The novelty of this study is divided into three parts. First, when the traditional assistance method is implemented in the power-assisted cart, we found that an OAR can be 
determined for each surface during stable walking via experimental verification. Second, we reveal the efficiency of the two proposed methods and also discuss their disadvantages. According to the experimental results, the scope of the application of each method is also discussed. Third, combining the two methods can overcome the mentioned drawbacks and maintain good performance while walking over a long distance. The results of this study could motivate manufacturers to improve similar existing devices on the market and also provide guidance on how to utilize the proposed methods for practical use.

\section{Specifications and Modeling of Power Assisted Cart: RT.1}

The experimental device, RT.1, is produced by Funai Electric Co. and RT.WORKS Co. (Figure 1a) and is now on sale in Japan [31]. The power-assisted cart can sustain the weight of the user and carry heavy loads during walking; the former decreases muscular activity, while the latter reduces the load-carrying burden. The number of cart users is dramatically increasing in Japan.

The specifications of the cart are reported in Table 1. There are force sensors inside the handles for measuring the force from the user, along the forward/backward direction only. The measured forces at the left and right handles are expressed as $F_{H L}$ and $F_{H R}$, respectively. Besides, the acceleration and angular velocity of the cart can be obtained via a built-in inertial measurement unit (IMU; MPU6050, TDK Invensense, San Jose, CA, USA). When the user pushes the handles of the power-assisted cart during walking (Figure 1b), two brushless DC motors at the rear wheels provide torque (driving force) for moving the cart. The rotational velocities of the two motors are acquired via hall effect sensors. The signal processing and control strategy are implemented by a microcontroller unit (RX63T, Renesas, Japan), of which the maximum operating frequency is up to $100 \mathrm{MHz}$. The frequency for the control loop was set as $500 \mathrm{~Hz}$.

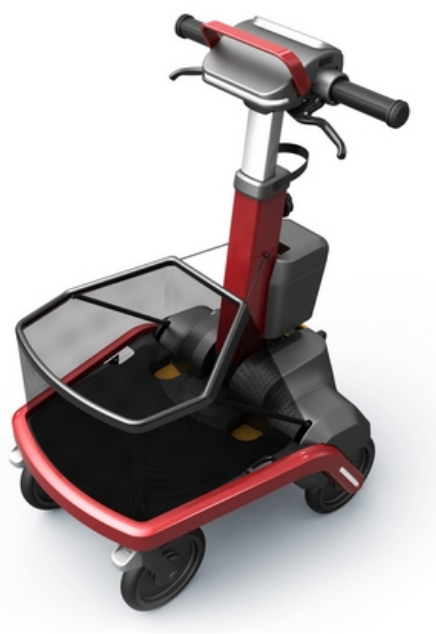

(a)

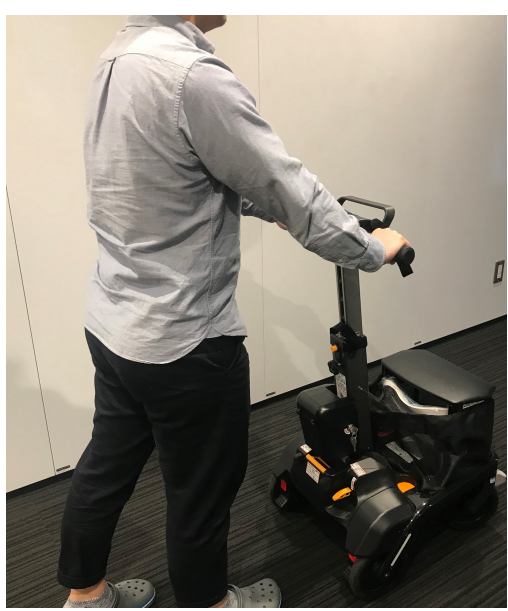

(b)

Figure 1. (a) Appearance and (b) experimental setup of the power-assisted cart RT. 1 provided by RT. WORKS [31].

Table 1. Specifications of RT.1.

\begin{tabular}{cc}
\hline Characteristic & RT.1 \\
\hline Dimensions & Width $510 \mathrm{~mm} \times$ length $601 \mathrm{~mm} \times$ height $819-1019 \mathrm{~mm}$ \\
\hline Weight & $14.8 \mathrm{~kg}$ \\
\hline Power & Lithium battery \\
\hline Mounted sensors & Force sensor, inertial measurement unit \\
\hline Microcontroller unit & RX63T, Renesas \\
\hline
\end{tabular}


As previously mentioned, the basic idea of such power-assisted devices for practical use can be summarized as the output for assistance is proportional to the input. For example, the cart enables the amount of assistance required to proceed and varies the steering depending on the input from the user. In this subsection, we describe the dynamic model of the cart with respect to the rotational center (the center of the rear wheels).

Figure 2a presents the model for the power-assisted cart. The front passive wheels can be considered as one caster for simplicity. The terms $F_{\text {Human }}$ and $M_{\text {Human }}$ are the resultant force and moment from the user applied to the rotational center, respectively; $F_{\text {Assist }}$ and $M_{\text {Assist }}$ are the resultant force and moment generated by two DC brushless motors to drive the rear wheels, respectively; and the forces generated by the left and right motors are represented by $F_{M L}$ and $F_{M R}$, respectively. The distance between the mass center and the rotational center is denoted as $l$. The relationship between the forces from the user and the two motors can be written as

$$
\left[\begin{array}{c}
F_{\text {Assist }} \\
M_{\text {Assist }}
\end{array}\right]=J_{1}\left[\begin{array}{c}
F_{\text {Human }} \\
M_{\text {Human }}
\end{array}\right] \text {, }
$$

where the matrix $J_{1}$ is equivalent to $\left[\begin{array}{cc}\alpha_{T} & 0 \\ 0 & \alpha_{R}\end{array}\right]$ and $\alpha_{T}$ and $\alpha_{R}$ are the ratios of the output to the input for translational and rotational movements, respectively. These forces and moments satisfy the following conditions:

$$
\begin{aligned}
{\left[\begin{array}{c}
F_{\text {Human }} \\
M_{\text {Human }}
\end{array}\right] } & =J_{2}\left[\begin{array}{c}
F_{H L} \\
F_{H R}
\end{array}\right], \\
{\left[\begin{array}{c}
F_{\text {Assist }} \\
M_{\text {Assist }}
\end{array}\right] } & =J_{3}\left[\begin{array}{c}
F_{M L} \\
F_{M R}
\end{array}\right],
\end{aligned}
$$

where $J_{2}=\left[\begin{array}{cc}1 & 1 \\ -r & r\end{array}\right]$ and $\boldsymbol{J}_{3}=\left[\begin{array}{cc}1 & 1 \\ -R & R\end{array}\right]$. Notations $r$ and $R$ express the displacements between one handle and the rotational center and between the rotational center and one of the wheels, respectively. Figure $2 \mathrm{~b}$ shows the resultant force and moment applied to the cart.
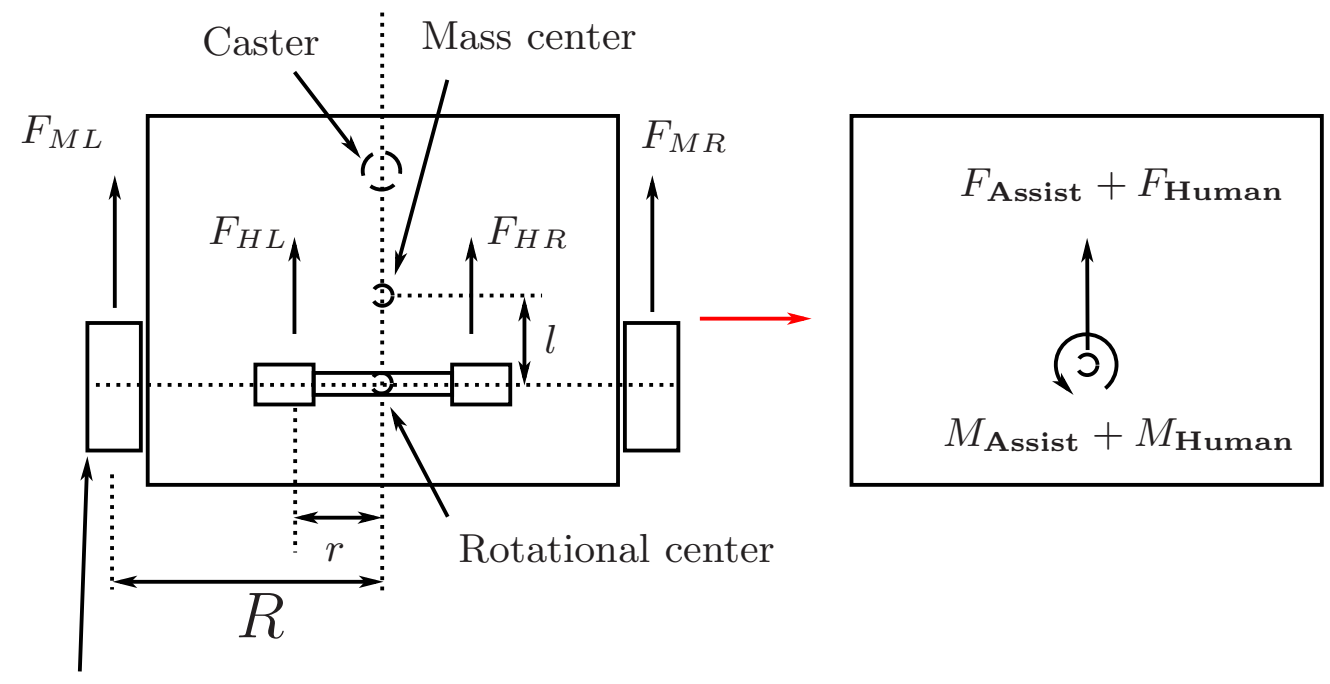

Rear wheel

(a)

Figure 2. (a) Model of the power-assisted cart and (b) resultant force and moment applied to the cart. 
Assistance ratios $\alpha_{T}$ and $\alpha_{R}$ are normally predefined as constants and their default values are set to be 1.5 and 0 , respectively, for RT.1. In this study, the motors assist both forward and rotational movements, and for simplicity, the ratio for forward movement is equivalent to the one for rotational movement, i.e., $\alpha_{T}=\alpha_{R}=\alpha$.

First, we introduce the equations of motion for the translational movement of the cart. We define $M, K_{v}$ and $g$ as the total mass of the cart and load, the equivalent viscous friction coefficient related to the translational movement, and gravitational acceleration, respectively. The translational velocities of both rear wheels are $v_{L}$ and $v_{R}$, respectively, and we define $v$ and $\omega$ as the translational and rotational velocities of the cart, respectively. It should be noted that

$$
\left[\begin{array}{c}
v \\
\omega
\end{array}\right]=J_{4}\left[\begin{array}{c}
v_{L} \\
v_{R}
\end{array}\right]
$$

where $\boldsymbol{J}_{4}=\left[\begin{array}{cc}\frac{1}{2} & \frac{1}{2} \\ -\frac{1}{2 R} & \frac{1}{2 R}\end{array}\right]=\boldsymbol{J}_{3}^{-T}$. When the cart moves on a slope as shown in Figure 3, the dynamics of the translational motion can be described as

$$
F_{T}=F_{\text {Human }}+F_{\text {Assist }}=M \frac{d v}{d t}+F_{v}+d_{T},
$$

where

$$
\begin{aligned}
F_{v} & =K_{v} v, \\
d_{T} & =M g(\mathrm{c} \beta \mathrm{s} \theta \mathrm{s} \gamma+\mathrm{s} \beta \mathrm{c} \gamma)+\mu_{c}\left(F_{N L}+F_{N R}\right) \mathrm{c} \beta c \theta,
\end{aligned}
$$

$\mu_{c}$ is the coefficient of kinetic friction in the translational direction, and $d_{T}$ expresses the disturbance including gravity and Coulomb friction. Notations $F_{N L}$ and $F_{N R}$ are for normal forces on the wheels, where $F_{N L}+F_{N R}=M g ; c *$ and $\mathrm{s} *$ mean $\cos (*)$ and $\sin (*)$, and $(\beta, \theta, \gamma)$ correspond to the Euler angles. It should be noted that $d_{T}$ is constant while moving on a flat surface or going straight on a slope whereas it will vary rapidly if the cart rotates on a slope.

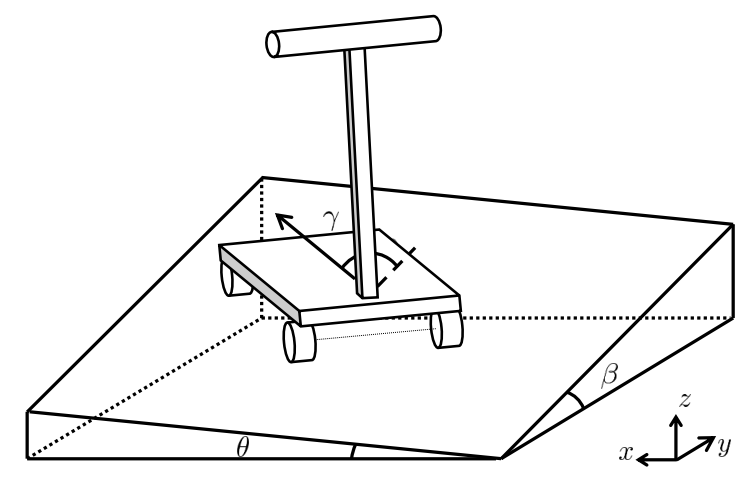

Figure 3. Movement of the cart on a slope.

Let $I$ be the rotational inertia of the cart and load with respect to the rotational center and $K_{\omega}$ be the coefficient of viscosity in rotational movement. Similarly, the dynamics of the cart related to rotational motion can be written as

$$
M_{T}=M_{\text {Human }}+M_{\text {Assist }}=I \frac{d \omega}{d t}+M_{w}+d_{R},
$$

where

$$
\begin{aligned}
M_{w} & =K_{\omega} \omega, \\
d_{R} & =l M g(-\mathrm{c} \beta \mathrm{s} \theta \mathrm{c} \gamma+\mathrm{s} \beta \mathrm{s} \gamma)+\mu_{c}\left(F_{N R}-F_{N L}\right) R \mathrm{c} \beta \mathrm{c} \theta,
\end{aligned}
$$


and $d_{R}$ represents the disturbance related to the rotational movement. If the disturbance can be ignored, the transfer functions related to translational and rotational movements are expressed as

$$
\begin{aligned}
\frac{v(s)}{F_{T}(s)} & =\frac{1}{M s+K_{v}}, \\
\frac{\omega(s)}{M_{T}(s)} & =\frac{1}{I s+K_{\omega}} .
\end{aligned}
$$

\section{Problem Formulation}

\subsection{Introduction to the Problem}

As mentioned above, assistance ratios $\alpha_{T}$ and $\alpha_{R}$ are commonly constant for commercial use. However, based on a preliminary study, it was found that DC motors with a fixed ratio cannot provide proper assistance in different environments because the friction term varies as the road surface changes. For example, the rolling resistance on sand is much larger than that on an indoor surface. Thus, if the assistance ratios of the cart are specified properly for indoor use, the user will feel that he or she needs extra force to operate the cart while walking on sand. In contrast, when the ratios are suitable for sand, the DC motor will offer excessive force while moving indoors. Consequently, the road roughness should be carefully dealt with for the daily use of a power-assisted cart.

During assisted walking, the operational force corresponds to the relative movement between the cart and the user. As the velocity of the user always fluctuates periodically during stable walking, the cart will also move in a similar manner. Figure 4 indicates the time histories of the operational forces during walking with the cart, which does not provide any assistance like a traditional walker. The subject (male, 28 years old, height $183 \mathrm{~cm}$, and weight $75 \mathrm{~kg}$ ) walked on an indoor surface, as is shown later. The data, including the operational forces and angular velocities, were recorded and filtered via a zero-phase 10th order low-pass filter with a cut-off frequency of $10 \mathrm{~Hz}$. The power spectral densities for the calculated resultant operational force $F_{\text {Human }}$ and translational velocity $v$ were estimated by using MATLAB function Periodogram, as shown in Figure 5. The same dominant frequency for $F_{\text {Human }}$ and $v$, which is related to the step time, was observed, thus the movement of the cart was governed by the user whose body accelerated and decelerated alternately during stable walking [32].

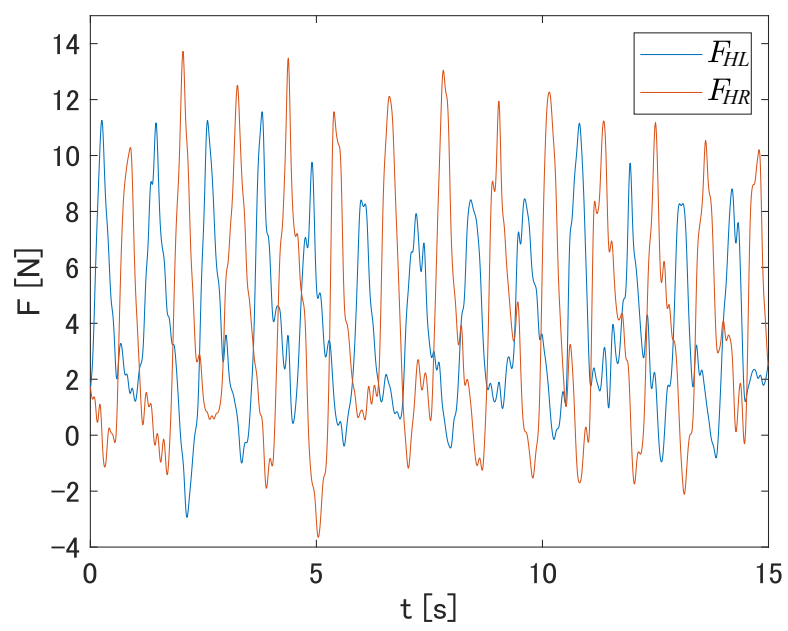

Figure 4. Operational forces for one subject with $\alpha=0$ in $15 \mathrm{~s}$ on the indoor surface.

Human-machine interaction also affects the movements of the user and the walking assistant during assisted walking. When a user walks steadily with the power-assisted cart, not only the operational forces but also the motor output exert an influence on the cart. The user must expend extra force to pull back the handles for slowing down the cart 
when its velocity becomes larger than the user. At this moment, the deceleration process is again amplified by $\alpha$, but the cart decelerates more than necessary when $\alpha$ is very large. Thus, in the next acceleration process, the uncoordinated movement between the cart and the user will also lead to a larger operational force. Finally, the overall operational force is increased, which brings about inefficiency and discomfort. On the other hand, with a small $\alpha$, the motor output slightly reduces the burden on the user. In conclusion, we hypothesize that there is an assistance ratio $\alpha$ that is most suitable for each surface; the hypothesis was verified, as is covered later on.

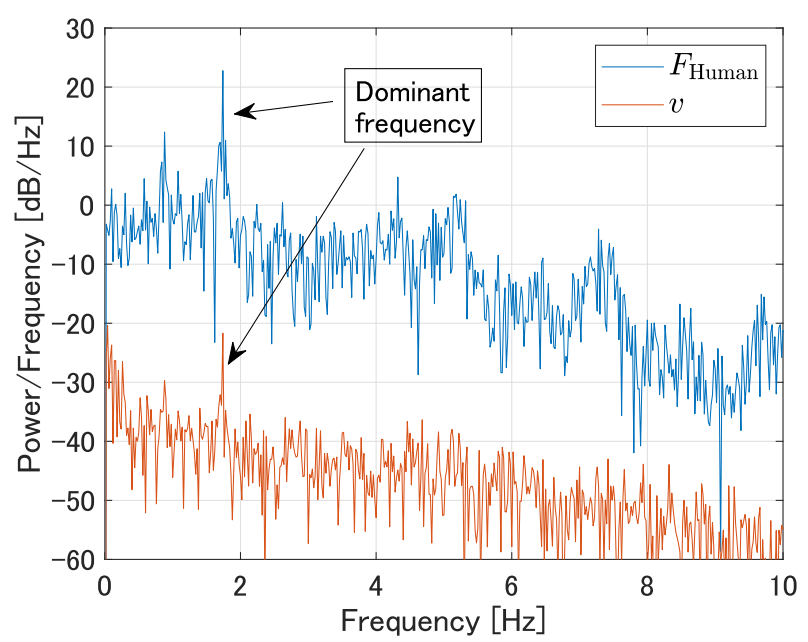

Figure 5. Estimated power spectral densities for resultant operational force $F_{\text {Human }}$ and translational velocity $v$.

\subsection{The Performance Index}

The following performance index was chosen to evaluate the operational efficiency to push the cart:

$$
W_{e}=\frac{\sum\left(\left|v_{L}\right| d T\right)+\sum\left(\left|v_{R}\right| d T\right)}{\sum\left(\left|F_{L} v_{L}\right| d T\right)+\sum\left(\left|F_{R} v_{R}\right| d T\right)},
$$

where $d T$ is the sampling time (0.01 s). The index was inspired by how the fuel efficiency of cars is measured, which is the distance traveled per unit volume of fuel used [33]. The numerator of $W_{e}$ is set to be the sum of the actual distances that the wheels move, which means that not only the translational movement but also the rotational one can be evaluated. When removing the norm, the denominator of $W_{e}$ becomes the work done by the user. When the operational force fluctuates dramatically (as discussed previously), the movement of the cart will disturb the gait of the user and make him or her feel uncomfortable. The occurrence of a large negative force is also not desirable when walking forward. Thus, we chose the absolute value of the work as the denominator of performance index $W_{e}$. A large value for $W_{e}$ implies less operational force and better efficiency to operate the cart. If an extreme point can be found on the curve, it is considered as the OAR. In addition, we also obtain the walking speed to quantify the gait ability during assisted walking. We propose two methods for solving the mentioned problems in the next section and verify the performance of the methods.

\subsection{Hypothesis Verification}

Four young healthy male subjects (age $26.62 \pm 1.42$ years old, height $171.14 \pm 8.37 \mathrm{~cm}$, and weight $68.53 \pm 3.59 \mathrm{~kg}$ ) participated in the pre-experiment to verify the hypothesis. The subjects operated the power-assisted cart on three types of flat surfaces, namely indoor, tile, and sand, as shown in Figure 6. On each surface, the subjects were asked to operate the cart to go straight forward for $20 \mathrm{~s}$ at four different walking speeds, while the assistance ratio of the cart $\left(\alpha_{T}\right)$ was set from 0 to 5 in increments of 1 and $\alpha_{R}=0$. Four different 
frequencies on a metronome were used during continuous walking to help the users maintain a steady speed or pace during assisted walking. Thus, there were $6 \times 4=24$ combinations of the assistance ratios and walking speeds for each surface, and five trials were conducted for each combination. Subsequently, the operational efficiency for each trial was calculated using (9).

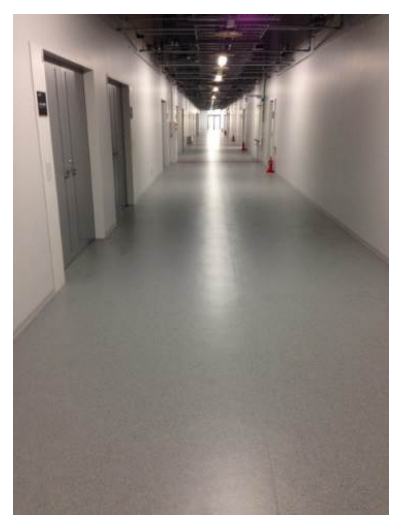

(a)

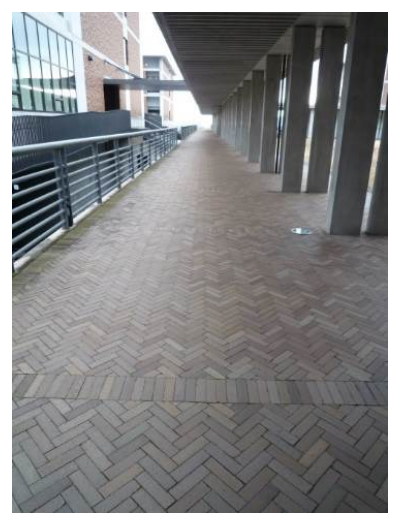

(b)

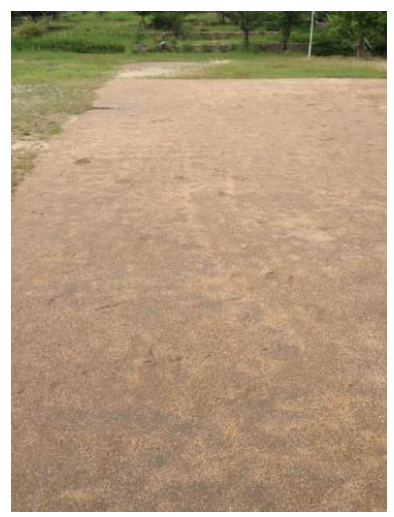

(c)

Figure 6. Experimental environments: (a) indoor, (b) tile, (c) sand.

Figure 7 shows the operational efficiency for the experimental trials of one subject on the indoor, tile, and sand surfaces. The asterisks illustrate the operational efficiency of the trials at different walking speeds, and the colored curves correspond to the curve fitting results. From the graphs, it is obvious that the extreme points of all curves fall in the intervals $[1.5,2]$ for indoor, $[3,4]$ for tile, and $[3,4]$ for sand. Moreover, similar results were observed for the other subjects. Therefore, we confirmed that there is an OAR with the highest efficiency for each kind of road surface. In addition, by averaging the experimental results, the values of 2.0 for indoor, 3.5 for tile, and 3.7 for sand are suitable for the OAR method.

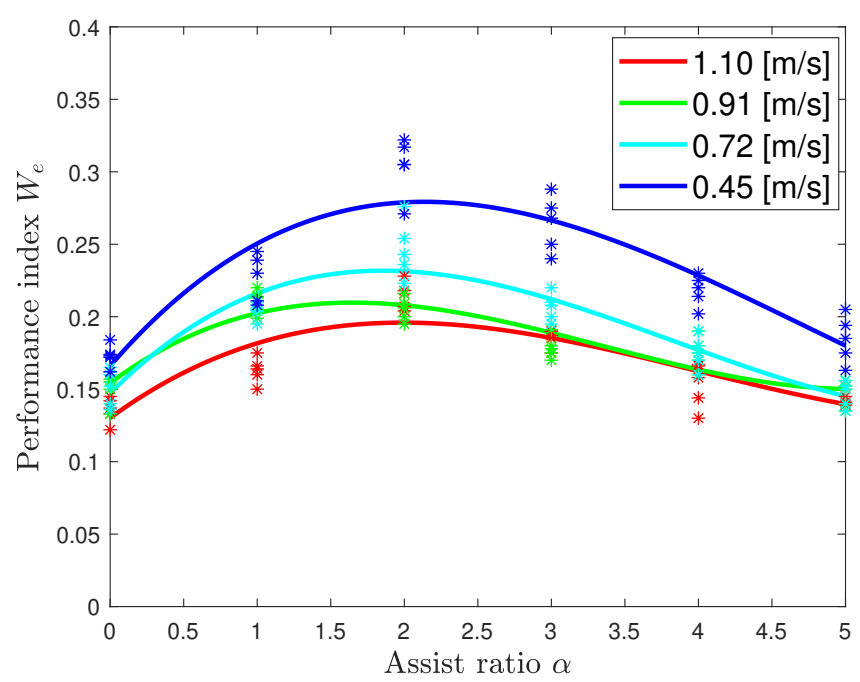

(a)

Figure 7. Cont. 


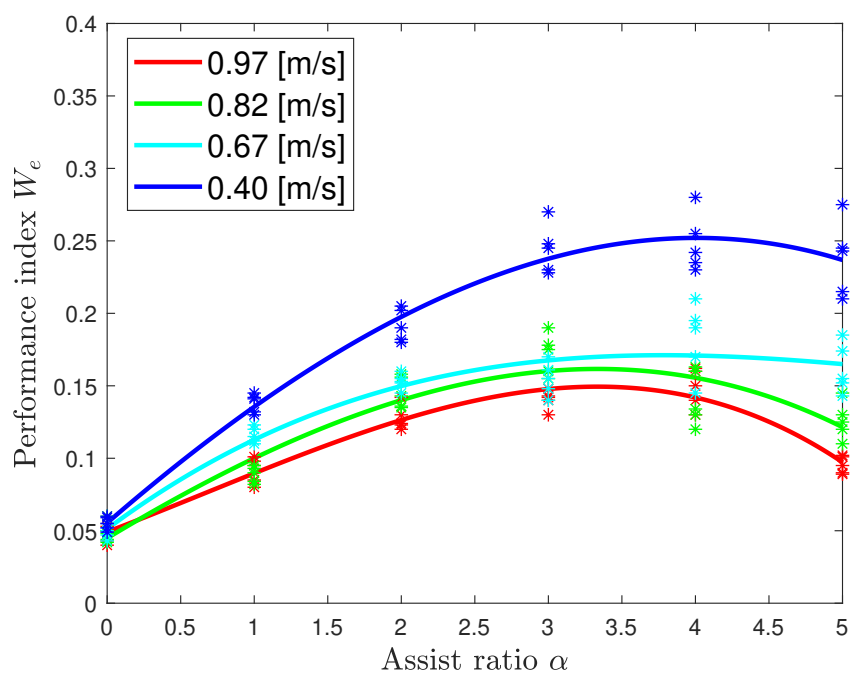

(b)

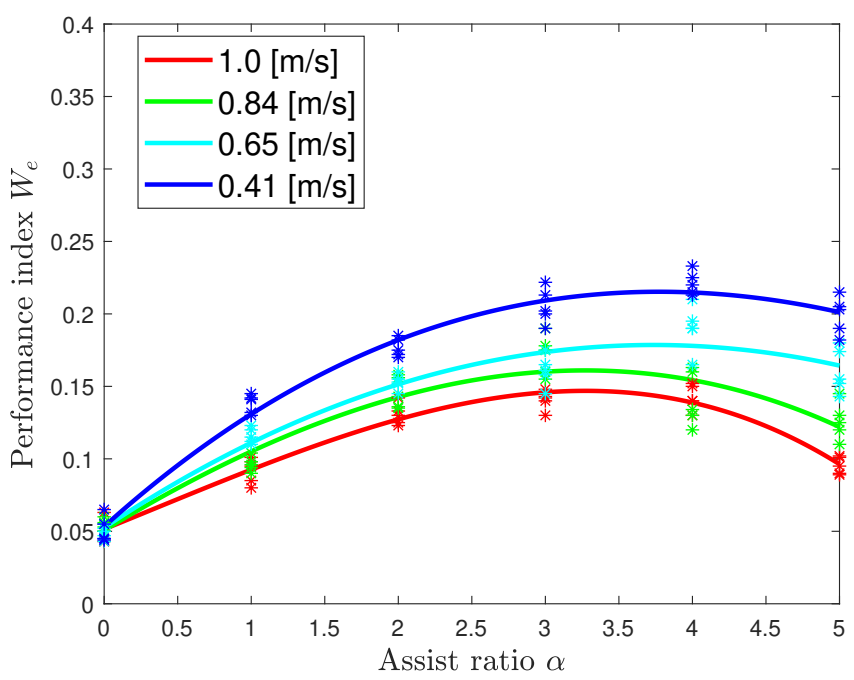

(c)

Figure 7. The relationship between operational efficiency and assistance ratio from the data of one subject on the (a) indoor, (b) tile, and (c) sand surfaces. The asterisks illustrate the operational efficiency of the trials at different walking speeds.

\section{Methods}

\subsection{The Oar Method}

The OAR method originates from the confirmed hypothesis. We consider that a properly selected assistance ratio will lead to driving forces suitable for each surface. If the current road surface is properly recognized during assisted walking, the user will be able to walk efficiently. Therefore, the cart must have the ability to recognize different terrain types. This can be accomplished by using machine learning technology, a typical procedure for which is as follows. First, raw data from driving the cart on different road types are collected. Second, the classifier is trained using features calculated from the raw data as input and using the road types as labels (target output). Third, the accuracy of the trained classifier is tested on new input data.

In this study, we used support vector machine (SVM) as the classifier and classified the surfaces into three groups: indoor, tile, and sand. First, acceleration in three axes $\left(a_{x}, a_{y}, a_{z}\right.$, corresponding to the lateral, forward, and vertical directions, respectively), translational velocity $v$, and resultant interaction force $F_{\text {Human }}$ were obtained at a frequency of $100 \mathrm{~Hz}$ from 
the IMU, hall effect sensors, and force sensors, respectively. Second, feature extraction was conducted due to the high sampling rate of the data and periodic fluctuation of human walking. In this study, the following features were selected as candidates:

$$
\begin{aligned}
& F_{1}=\max \left(x_{i}\right), \\
& F_{2}=\frac{1}{N} \sum_{i=1}^{N} x_{i}, \\
& F_{3}=\frac{1}{N} \sum_{i=1}^{N}\left(x_{i}-F_{2}\right)^{2}, \\
& F_{4}=\exp \left(\frac{1}{N} \sum_{i=1}^{N} \log \left|x_{i}\right|\right), \\
& F_{5}=\frac{1}{N-1} \sum_{i=1}^{N-1}\left|x_{i+1}-x_{i}\right|,
\end{aligned}
$$

where $x_{i}$ is the $i$ th data item and $N$ is the number of data items in the time window. The time window is set to 1 s, i.e., $N=100$. The features correspond to the Maximum, Average, Mean Square Error, Log Detector, and Average Amplitude Change of the data, respectively. The offline training of the SVM was conducted using MATLAB ver. 9.0 (Mathworks, Natick, MA, USA). After the training, the SVM classifier was implemented in the power-assisted cart.

After considering the limited performance of the cart-mounted motor control unit (MCU) and the trade-off between the classification accuracy and computational load, we did not use all of the sensor data and features. Instead, we surveyed the sensor data items and features and tried to determine the most effective combination. More details are provided in Section 5.

\subsection{The DOB Method}

This is proposed for estimating and canceling disturbance (as explained in Section 2) and is applied to both the translational and rotational motions of the cart. To utilize the disturbance observer, the terms in (4) and (6) are considered as time-persistent disturbances, i.e., $\dot{d}_{T}=\dot{d}_{R}=0$. The low-pass filter plays a significant role in DOB, and it takes time to compensate for the disturbance due to the phase delay. While rotating on a slope, the disturbance varies rapidly as the cart rotates, resulting in the conditions $\dot{d}_{T} \neq 0$ and $\dot{d}_{R} \neq 0$. Thus, the movement of rotating on a slope was not covered in this study.

Figure 8 shows a block diagram of the whole system, where $P_{1}, P_{2}, Q_{1}$, and $Q_{2}$ are the real plants and low-pass filters for the translational and rotational movements, respectively. Different from the traditional method, a low-pass filter of $\frac{1}{0.1 s+1}$ was used to make the inputs of the disturbance observer, such as force and velocity smoothing. Since it is impossible to measure the weights of the load and friction in daily life accurately, the parameters for the real plants $P_{1}$ and $P_{2}$ are unknown but limited. As mentioned in [34], the behavior of a real system can be made similar to the nominal one by using the disturbance observer. Apart from the time-persistent disturbances $d_{T}$ and $d_{R}$, the DOB method also estimates and cancels the difference between the real plant and the nominal one. The design of the low-pass filter in DOB is extremely significant, and the filter was chosen as

$$
Q_{*}(s)=\frac{1}{\tau_{*} s+1}, *=1,2,
$$

where $\tau_{*}$ corresponds to the cut-off frequency (a small $\tau_{*}$ leads to a fast response). It should be noted that a response that is too fast may make the user feel uncomfortable, and thus, different values of $\tau_{1}$ and $\tau_{2}$ for the translational and rotational movements need to be properly selected. In this study, the value of $\tau_{1}$ brought about a faster response than that of $\tau_{2}$. 


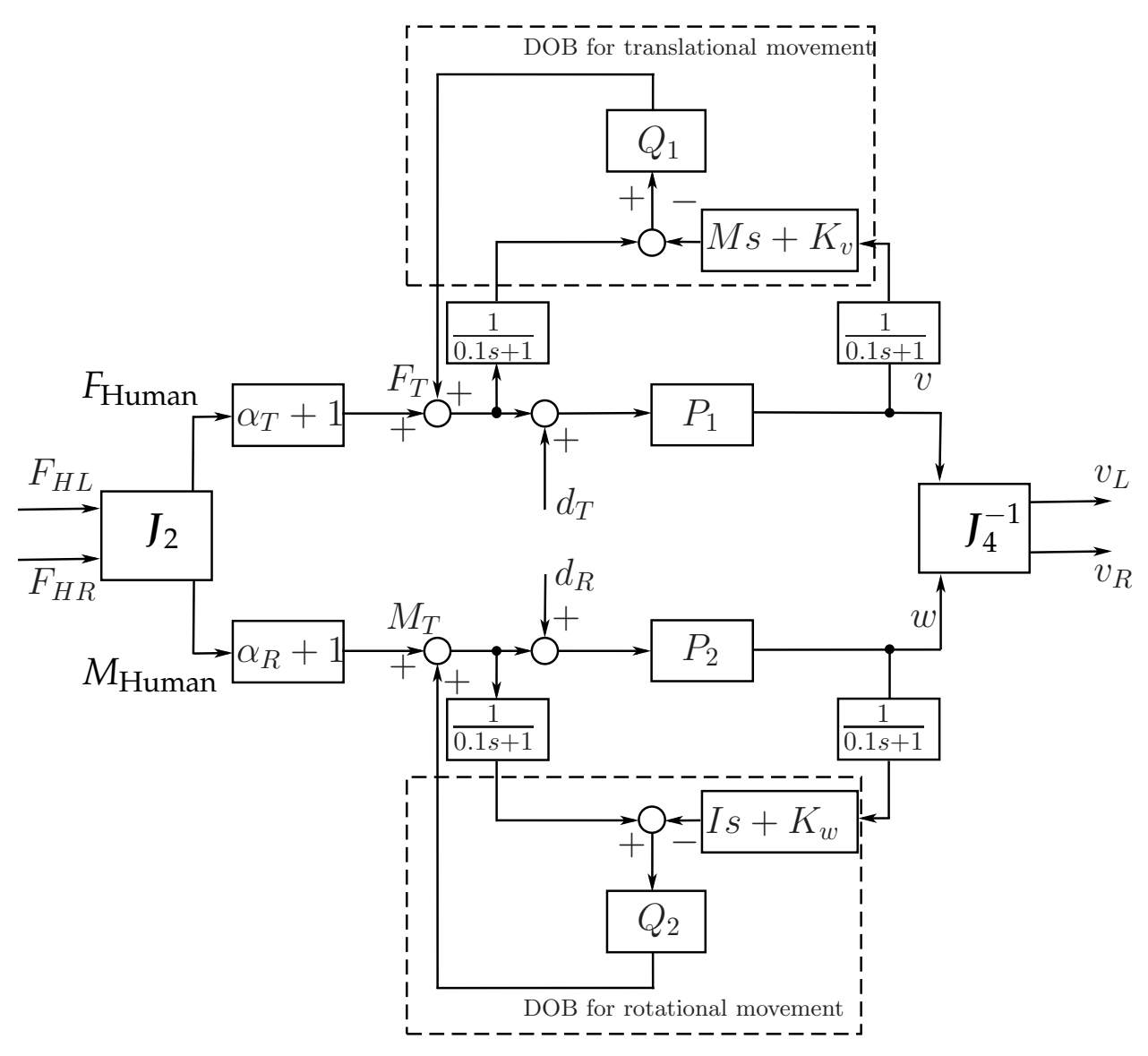

Figure 8. A block diagram of the cart.

\section{Experiment}

Experiments were carried out to evaluate the validity of the proposed methods. Here, the details of the experiments and the experimental results are reported. First, pre-experiments were carried out to determine the features and inputs for OAR and the parameters for DOB. Second, the OAR and DOB methods were applied for movement on flat surfaces and a slope, respectively.

\subsection{Pre-Experiment}

\subsubsection{Feature Selection}

As mentioned before, we tried to find effective combinations for reducing the burden of the cart-mounted MCU, from using 5 features $\left(F_{1}, \ldots, F_{5}\right)$ and 5 inputs: accelerations of the cart $\left(a_{x}, a_{y}, a_{z}\right)$, the resultant force $\left(F_{\text {human }}\right)$, and the translational velocity $(v)$. For a certain combination, the features related to each set of sensor data items were calculated for each combination of inputs .

Table 2 summarizes the details of the five most effective combinations. Combination D resulted in high accuracy and had a relatively small number of inputs. In comparison to Combination $\mathrm{D}$, the deficiency of the acceleration $a_{y}$ in Combination $\mathrm{E}$ resulted in a rapid reduction in accuracy for sand. As we know, the computational load will increase with the number of inputs when calculating the input for an SVM. Since the performance of the cart-mounted MCU is limited, we need to maintain the balance between high classification accuracy and low computational load. Thus, we chose Combination D as the input for the SVM embedded in the power-assisted cart. 
Table 2. The input combinations for SVM and the corresponding accuracy.

\begin{tabular}{|c|c|c|c|c|c|c|}
\hline \multicolumn{2}{|c|}{ Combination } & $\mathbf{A}$ & B & $\mathrm{C}$ & D & $\mathrm{E}$ \\
\hline \multirow{3}{*}{ Feature } & $F_{2}$ & $\checkmark$ & $\checkmark$ & $\checkmark$ & $\checkmark$ & $\checkmark$ \\
\hline & $F_{3}$ & $\checkmark$ & $\checkmark$ & & & \\
\hline & $F_{5}$ & $\checkmark$ & $\checkmark$ & $\checkmark$ & $\checkmark$ & $\checkmark$ \\
\hline \multirow{5}{*}{ Sensor } & $a_{y}$ & $\checkmark$ & $\checkmark$ & $\checkmark$ & $\checkmark$ & \\
\hline & $a_{x}$ & $\checkmark$ & $\checkmark$ & $\checkmark$ & $\checkmark$ & $\checkmark$ \\
\hline & $a_{z}$ & $\checkmark$ & & $\checkmark$ & & \\
\hline & $v$ & $\checkmark$ & $\checkmark$ & $\checkmark$ & $\checkmark$ & $\checkmark$ \\
\hline & $F_{\text {human }}$ & $\checkmark$ & $\checkmark$ & $\checkmark$ & $\checkmark$ & $\checkmark$ \\
\hline \multicolumn{2}{|c|}{ Number of input } & 15 & 12 & 10 & 8 & 6 \\
\hline \multicolumn{2}{|c|}{ Training accuracy [\%] } & 99.5 & 98.5 & 98.2 & 97.36 & 95.4 \\
\hline \multicolumn{2}{|c|}{ Test accuracy [\%] } & 97.7 & 97.3 & 96.1 & 95.3 & 93.3 \\
\hline \multicolumn{2}{|c|}{ Indoor [\%] } & 99.5 & 99.6 & 99.8 & 99.7 & 99.5 \\
\hline \multicolumn{2}{|c|}{ Tile [\%] } & 96.5 & 95.6 & 93.7 & 92.8 & 90.0 \\
\hline \multicolumn{2}{|c|}{ Sand [\%] } & 96.1 & 95.1 & 92.3 & 90.0 & 85.8 \\
\hline
\end{tabular}

\subsubsection{Parameter Selection for DOB}

The physical parameters of the power-assisted cart were obtained through parameter identification as $M=14.8, K=1.68, I=0.095$ and $K_{\omega}=0.16$. The experiment was conducted without an external load in the indoor environment (Figure 6a). These parameters are set to be the ones for the nominal plant of DOB. Moreover, the parameters of the low-pass filters used in DOB are were chosen as $\tau_{1}=0.1$ for the forward movement and $\tau_{2}=0.5$ for the rotational movement. All of the subjects reported that they could walk without feeling uncomfortable.

When DOB is activated, the cart can stop on a slope at any angle $\gamma$, as shown in Figure 9. The proposed disturbance observer cancels the effect of gravity and prevents the cart from slipping down the slope. With a simple DOB which that only compensates for the disturbance related to the translational movement, the cart can only stop on a slope with $\gamma=0$ because of the gravitational effect on the rotational movement.

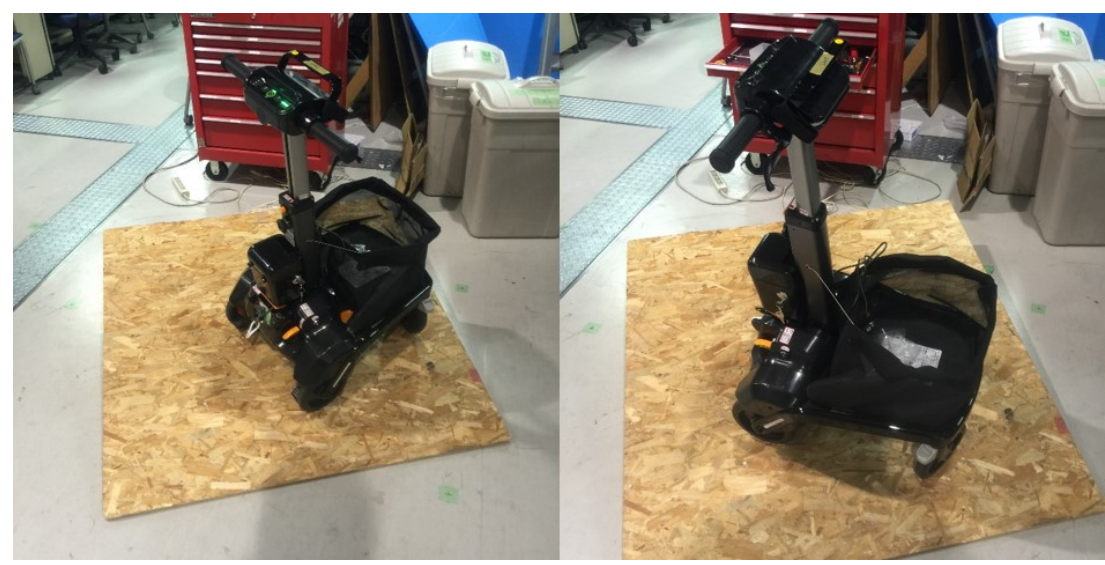

Figure 9. Photographs showing that the cart can stop on a slope in at any attitude angle (Specification specifications of the slope: $\theta=0^{\circ}, \beta=10^{\circ}$, wood) when a disturbance observer-based (DOB) method is activated.

\subsection{Experimental Setup}

Eight healthy male subjects (age $25.38 \pm 1.77$ years, height $171.75 \pm 7.40 \mathrm{~cm}$, and weight $69.88 \pm 3.87 \mathrm{~kg}$ ) who consented to the experimental protocol approved by the Institutional 
Review Board of Kyoto University, participated in the experiment. None of them had any history of injuries to their lower extremities or of falling in the past year. Data during walking with OAR and DOB, without assistance $(\alpha=0)$, and with the default assistance ratio were obtained for comparison. The default assistance ratio was the same as that used in products already on the market, i.e., $\alpha=1.5$. Ratio $\alpha$ was set as 1.5 for DOB and the relevant value for the particular environment obtained in the pre-experiment of Section 3.3 for OAR. In the cases with the default assistance ratio and OAR, the gravity compensation from the motors just counteracted the gravity effect of the cart excluding the weight of the load. Moreover, since we wanted to determine the robustness of each method to changes in load weight, the experiment was conducted with and without a load of $10 \mathrm{~kg}$.

The experiment was conducted on three flat surfaces and a slope, as shown in Figures 6 and 10. The slope of 8 degrees and the tile surface are of the same material. In the flat surface experiment, the subjects moved with the cart along a straight line and in a circle of $1 \mathrm{~m}$ radius, while they only moved straight up a slope with $\gamma=0$ in the slope experiment. In the straight-line walking cases, the subjects walked for $15 \mathrm{~s}$, which was repeated five times. In the circular walking cases, the subjects moved along the circle for $50 \mathrm{~s}$. For all of the trials, the step frequency during walking was set by using a metronome at a frequency of $85 \mathrm{bpm}$. The participants could walk slowly at a speed of equal or less than $0.8 \mathrm{~m} / \mathrm{s}$ to simulate the locomotion of the frail elderly [35].

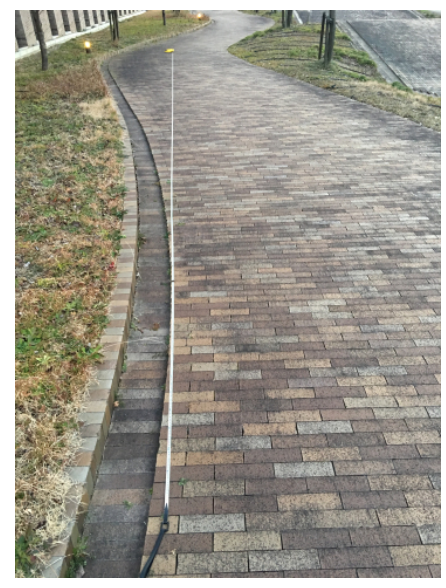

Figure 10. Experimental environment: slope of 8 degrees and the same material as the tile surface.

\subsection{Data Processing and Statistical Analysis}

After each trial, the performance index $W_{e}$ in (9) was calculated. Moreover, we averaged the performance indices for 5 repetitions from the data of each participant during straight-line walking. Walking speed was affected by the step length because of the walking constraint induced by the metronome, and so to cancel out the difference between individuals, the dimensionless walking speed $V_{D}$ was obtained by scaling to the leg length $L$ of each participant, as suggested in [36]: $V_{D}=v / \sqrt{g L}$. Data processing and statistical analysis, including the Kruskal-Wallis test (nonparametric one-way analysis of variance (ANOVA)) and multiple comparison tests, were conducted in MATLAB, while nonparametric two-way ANOVA was performed with $\mathrm{R}$ based on the Aligned Rank Transform [37]. For the oneway ANOVA, the effect of the control method on the dimensional walking speed for each surface was investigated. For the two-way ANOVA on operational efficiency, the surfaces and control methods with/without the load were considered as factors to be examined. Multiple comparisons were performed if there was a statistically significant difference in a particular test. A $p$-value of less than 0.05 was considered statistically significant. Noise in the experimental data was removed through applying a zero-phase 10th order low-pass filter with a cut-off frequency of $10 \mathrm{~Hz}$. 


\subsection{Experimental Results}

Table 3 reports the averaged operational efficiency without a load for each condition and surface. The results of nonparametric two-way ANOVA for the operational efficiency show statistically significant differences between the surfaces $(F=128.61, p<0.01)$ and between the methods $(\mathrm{F}=16.11, p<0.01)$, while interaction between these factors was also observed $(\mathrm{F}=3.48, p<0.01)$. Due to the significant differences, multiple comparisons tests for the methods were performed. The averaged operational efficiencies for OAR and DOB were significantly different from the ones for the case without assistance and with the default method, while there was no evidential difference between OAR and DOB.

The averaged operational efficiency with load of $10 \mathrm{~kg}$ for each condition and surface is reported in Table 4. The results of nonparametric two-way ANOVA for the operational efficiency indicate statistically significant differences between the surfaces $(F=202.15$, $p<0.01)$ and between the methods $(\mathrm{F}=52.47, p<0.01)$, while interaction between these factors was also observed $(\mathrm{F}=5.45, p<0.01)$. The results of multiple comparison tests for the methods illustrate a significant difference between DOB and the other methods.

The results of nonparametric one-way ANOVA and multiple comparison tests for the dimensionless walking speed indicate that there were almost no significant differences in the dimensionless walking speed between the four methods for all of the surfaces, except that the dimensionless walking speed for DOB $\left(V_{D}=0.26\right)$ was significantly different from the ones for the case without assistance $\left(V_{D}=0.20\right)$ and with default assistance $\left(V_{D}=0.20\right)$ while walking upon the slope $(p=0.016$ without the load and $p<0.01$ with the load).

Table 3. Averaged operational efficiency without load.

\begin{tabular}{cccccc}
\hline Environment & Method & No Assistance & Default & OAR & DOB \\
\hline \multirow{3}{*}{$\begin{array}{c}\text { Flat } \\
\text { Straight }\end{array}$} & Indoor & 0.319 & 0.505 & 0.539 & 0.504 \\
\cline { 2 - 5 } & Tile & 0.327 & 0.461 & 0.544 & 0.552 \\
\cline { 2 - 5 } Flat & Sand & 0.166 & 0.321 & 0.450 & 0.591 \\
\cline { 2 - 6 } Circular & Indoor & 0.272 & 0.520 & 0.609 & 0.557 \\
\cline { 2 - 6 } & Tile & 0.287 & 0.439 & 0.619 & 0.516 \\
\hline Slope & Sand & 0.179 & 0.350 & 0.455 & 0.538 \\
Straight & Upwards & 0.052 & 0.361 & 0.461 & 0.555 \\
\cline { 2 - 6 } & Downwards & 0.111 & 0.381 & 0.458 & 0.458 \\
\hline
\end{tabular}

Table 4. Averaged operational efficiency with a load of $10 \mathrm{~kg}$.

\begin{tabular}{cccccc}
\hline Environment & Method & No Assistance & Default & OAR & \multirow{2}{*}{ DOB } \\
\hline \multirow{3}{*}{$\begin{array}{c}\text { Flat } \\
\text { Straight }\end{array}$} & Indoor & 0.273 & 0.468 & 0.476 & 0.416 \\
\cline { 2 - 6 } & Tile & 0.306 & 0.421 & 0.486 & 0.540 \\
\cline { 2 - 6 } Flat & Sand & 0.110 & 0.249 & 0.392 & 0.552 \\
\cline { 2 - 6 } Circular & Indoor & 0.197 & 0.350 & 0.367 & 0.388 \\
\cline { 2 - 6 } Slope & Tile & 0.161 & 0.277 & 0.415 & 0.487 \\
Straight & Sand & 0.119 & 0.214 & 0.347 & 0.461 \\
\cline { 2 - 6 } & Upwards & 0.038 & 0.141 & 0.212 & 0.352 \\
\hline
\end{tabular}




\section{Discussion}

From the results of the experiments in Section 3.3, it was found that there was a maximum operational efficiency for each surface and walking speed during stable walking, which demonstrates the validity of the hypothesis. According to the data from the four subjects, OAR was chosen as 2.0 for indoor, 3.5 for tile, and 3.7 for sand, respectively. Hence, the power-assisted cart needs to provide more assistance in a complex environment. In addition, we found that the performance index decreased with an increment in walking speed.

Our experimental results also reveal that the operational efficiency values for the two proposed methods were relatively better than the ones for no assistance and default assistance. The OAR method had better operational efficiency for the indoor flat road, while DOB provided the most efficient assistance in most of the other cases. On the other hand, the improvement in efficiency with the DOB method resulted from the compensation to counteract disturbance and reducing the necessary force for pushing the cart, especially on a slope. However, with a load of $10 \mathrm{~kg}$ in the indoor environment, the operational efficiency for DOB was no better than that for the default assistance of $\alpha=1.5$. This could be the result of using the low-pass filter since the difference between the actual plant and the nominal one was relatively small. In the experiments of this study, young subjects walked slowly to simulate the locomotion of the elderly. Since the step frequency was fixed in this study with the use of a metronome, increased walking speed corresponded to an increment in step length. The difference in dimensionless walking speed while walking upon the slope indicates that if DOB is active, the cart could easily be pushed even with a heavy load.

The time histories of the operational forces for one trial with DOB with the same surface and participant as in Figure 4 without assistance is shown in Figure 11. The periodic variation related to gait became indistinct in comparison with that in Figure 4, while the necessary force to push the cart was drastically reduced. This implies that the force to move the cart was not only generated by the subject but also by the motor output.

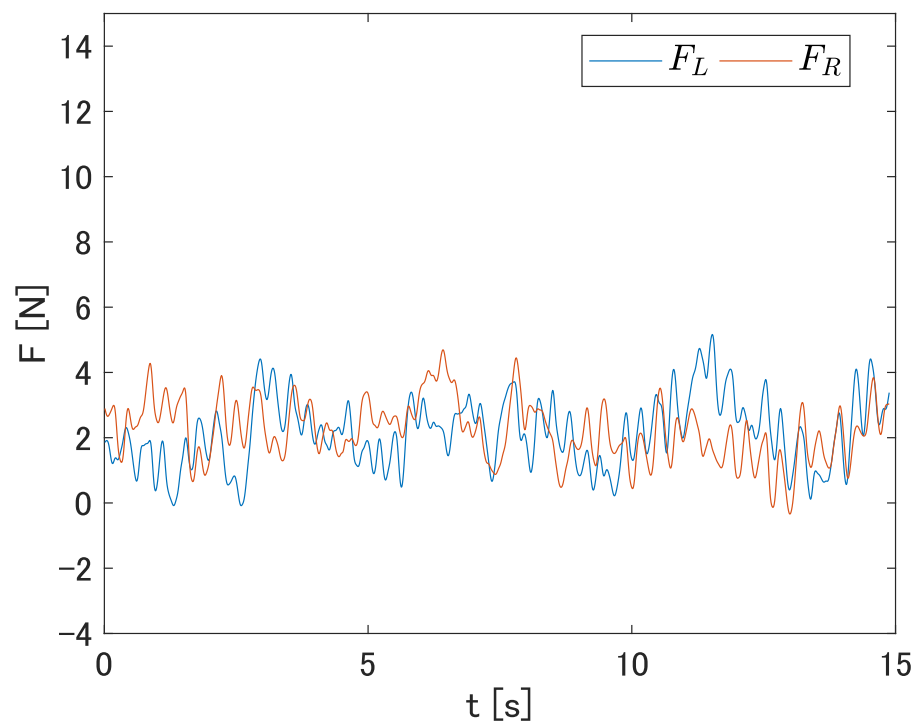

Figure 11. Operational forces of one subject with DOB in $15 \mathrm{~s}$ on an indoor surface.

Both methods have some drawbacks. As shown in Section 3, the operational efficiency could be considered as a unimodal function of $\alpha$ for a specific surface and walking speed, while the offline procedure in Section 5 for obtaining the OAR was very complex. Instead, the optimal one could be acquired via an on-line approach, and a golden-section search would be useful due to the relationship between the operational efficiency and assistance ratio. Moreover, the movement of walking on an unknown surface might result in high 
classification error and improper assistance from the cart, a problem that could be solved by simplifying the categories for classification.

In addition, there is still a serious limitation while utilizing DOB. It was found that with $\mathrm{DOB}$, when the user rotated on a slope, the cart became extremely hard to operate due to the rapid change in the disturbance. Thus, rotational movement on a slope needs to be detected and DOB must be turned off during rotation.

In conclusion, each method has its merits and demerits. It seems better to utilize DOB in an unknown environment and OAR for indoor surfaces. The way of combining both methods may become accepted as a suitable strategy for solving the above problems. First, the surface is classified via a machine learning approach such as an SVM into only two categories: indoor and outdoor. The rotational movement and posture of the cart are detected simultaneously, then a proper method can be chosen according to the classification and detection results. For instance, the cart can be controlled by applying the OAR method for the indoor flat surface and switching to the DOB method for outdoor use.

We combined OAR and DOB and implemented the above algorithm. Acceleration data and rotational velocities were processed for the detection of posture and rotational movement. The combined method and default assistance ratio were included for comparison. One subject walked freely for $6 \mathrm{~min}$ with the power-assisted cart and load of $10 \mathrm{~kg}$. The experimental space included but was not limited to the surfaces in Figure 6. The classification rate of $98.4 \%$ indicates high accuracy. The obtained mean operational efficiency for the combined method is 0.502 , while the one for the default assistance ratio is just 0.317. In addition, since DOB was turned off, the user did not feel uncomfortable while rotating on the slope. The result indicates the high performance of the combined method.

\section{Conclusions}

In this paper, two methods of controlling a power-assisted cart have been proposed for practical use. The OAR method searches for the optimal assistance ratios on different types of roads, and then a classifier is integrated to recognize the road type based on SVM. Through pre-experimental verification, it established an OAR for each surface during stable walking. The DOB method estimates and cancels the disturbance with respect to the environmental effect. Eight healthy subjects participated in an experiment of cart-assisted walking on different flat road surfaces and a slope. The experimental results indicate higher efficiency of OAR and DOB methods while walking with the cart on different surfaces compared to the cases without assistance and with default assistance. Especially on a slope, DOB led to the best performance with high efficiency and steady walking speed. The drawbacks of the proposed methods and how to overcome them by sole use or in combination were discussed: the cart can be controlled with high performance by using OAR during walking indoors and DOB during walking outdoors, while their drawbacks could be eliminated by combining them. The findings of this study provide guidance on how to utilize the proposed methods for practical use.

Author Contributions: Conceptualization, X.W. and F.M.; methodology, X.W. and J.M.; software, X.W., J.M. and Y.Z.; validation, X.W., T.E. and F.M.; formal analysis, X.W.; investigation, X.W., J.M. and Y.Z.; resources, X.W.; data curation, X.W. and T.E.; writing-original draft preparation, X.W.; writing-review and editing, T.E. and F.M.; visualization, X.W.; supervision, T.E. and F.M. ; project administration, F.M.; funding acquisition, F.M. All authors have read and agreed to the published version of the manuscript.

Funding: This research received no external funding.

Institutional Review Board Statement: The study was approved by the Institutional Review Board of Kyoto University.

Informed Consent Statement: Informed consent was obtained from all subjects involved in the study.

Conflicts of Interest: The authors declare no conflict of interest. 


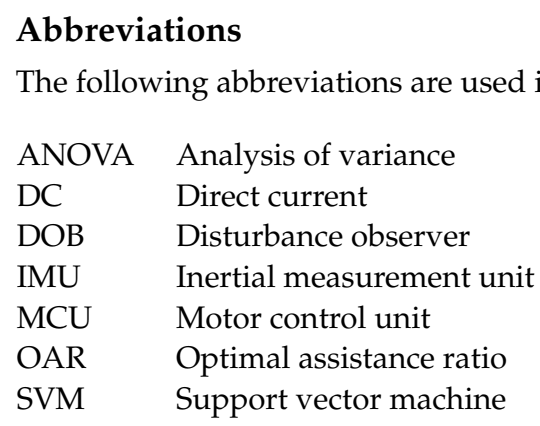

\section{References}

1. Current Population Estimates as of October 1, 2014. Available online: http://www.stat.go.jp/english/data/jinsui/2014np/ index.htm (accessed on 25 November 2020).

2. Esquenazi, A.; Talaty, M.; Packel, A.; Saulino, M. The ReWalk powered exoskeleton to restore ambulatory function to individuals with thoracic-level motor-complete spinal cord injury. Am. J. Phys. Med. Rehabil. 2012, 91, 911-921. [CrossRef]

3. Reis, L.P.; Braga, R.A.; Sousa, M.; Moreira, A.P. IntellWheels MMI: A flexible interface for an intelligent wheelchair. In Proceedings of the Robot Soccer World Cup, Graz, Austria, 29 June-5 July 2009; Springer: Berlin/Heidelberg, Germany, 2009 ; pp. $296-307$.

4. Spenko, M.; Yu, H.; Dubowsky, S. Robotic personal aids for mobility and monitoring for the elderly. IEEE Trans. Neural Syst. Rehabil. Eng. 2006, 14, 344-351. [CrossRef]

5. Maeda, H.; Fujiwara, S.; Kitano, H.; Yamashita, H.; Fukunaga, H. Control of an Omni-directional Power-assisted Cart. JSME Int. J. Ser. C Mech. Syst. Mach. Elem. Manuf. 2003, 46, 931-937. [CrossRef]

6. Hara, S. A smooth switching from power-assist control to automatic transfer control and its application to a transfer machine. IEEE Trans. Ind. Electron. 2007, 54, 638-650. [CrossRef]

7. Wakabayashi, Y.; Seino, A.; Kinugawa, J.; Kosuge, K. Design and control of a heavy-load, low-cost power-assisted cart using brakes and a differential gear system. In Proceedings of the IEEE International Conference on Advanced Intelligent Mechatronics (AIM), Munich, Germany, 3-7 July 2017; pp. 4-9.

8. Lee, G.; Ohnuma, T.; Chong, N.Y. Design and control of JAIST active robotic walker. Intell. Serv. Robot. 2010, 3, 125-135. [CrossRef]

9. Fontanelli, D.; Giannitrapani, A.; Palopoli, L.; Prattichizzo, D. A passive guidance system for a robotic walking assistant using brakes. In Proceedings of the IEEE Conference on Decision and Control (CDC), Osaka, Japan, 15-18 December 2015; pp. 829-834.

10. Ko, C.H.; Young, K.Y.; Huang, Y.C.; Agrawal, S.K. Active and passive control of walk-assist robot for outdoor guidance. IEEE/ASME Trans. Mechatron. 2013, 18, 1211-1220. [CrossRef]

11. Paulo, J.; Peixoto, P.; Nunes, U.J. ISR-AIWALKER: Robotic walker for intuitive and safe mobility assistance and gait analysis. IEEE Trans. Hum.-Mach. Syst. 2017, 47, 1110-1122. [CrossRef]

12. Chalvatzaki, G.; Papageorgiou, X.S.; Maragos, P.; Tzafestas, C.S. User-Adaptive Human-Robot Formation Control for an Intelligent Robotic Walker using Augmented Human State Estimation and Pathological Gait Characterization. In Proceedings of the IEEE/RSJ International Conference on Intelligent Robots and Systems, Madrid, Spain, 1-5 October 2018; pp. 6016-6022.

13. Kawamoto, H.; Lee, S.; Kanbe, S.; Sankai, Y. Power assist method for HAL-3 using EMG-based feedback controller. In Proceedings of the IEEE International Conference on Systems, Man and Cybernetics, Washington, DC, USA, 8 October 2003; Volume 2, pp. 1648-1653.

14. Kiguchi, K.; Hayashi, Y. An EMG-based control for an upper-limb power-assist exoskeleton robot. IEEE Trans. Syst. Man Cybern. Part B (Cybern.) 2012, 42, 1064-1071. [CrossRef]

15. Lenzi, T.; De Rossi, S.M.M.; Vitiello, N.; Carrozza, M.C. Intention-based EMG control for powered exoskeletons. IEEE Trans. Biomed. Eng. 2012, 59, 2180-2190. [CrossRef]

16. Yu, H.; Spenko, M.; Dubowsky, S. An adaptive shared control system for an intelligent mobility aid for the elderly. Auton. Robot. 2003, 15, 53-66. [CrossRef]

17. Kobayashi, N.; Tanaka, T.; Kaneko, S. Power-Assisted Cart Control Method with Motor Torque Limiter Based on Prediction of Torque Saturation. J. Energy Power Eng. 2012, 6, 1349.

18. Weiss, C.; Tamimi, H.; Zell, A. A combination of vision-and vibration-based terrain classification. In Proceedings of the IEEE/RSJ International Conference on Intelligent Robots and Systems, Nice, France, 22-26 September 2008; pp. 2204-2209.

19. Watanabe, T.; Katsura, S. On-line recognition of driving road condition using support vector machine. In Proceedings of the IEEE International Conference on Industrial Technology, Auburn, AL, USA, 14-16 March 2011; pp. 405-410.

20. Wang, S.; Kodagoda, S.; Khushaba, R. Towards speed-independent road-type classification. In Proceedings of the International Conference on Control Automation Robotics \& Vision (ICARCV), Guangzhou, China, 5-7 December 2012; pp. 614-619.

21. Yokoyama, Y. Bicycle with Assist Engine. U.S. Patent 5,937,962, 17 August 1999.

22. Nagai, H.; Tomita, K.; Tsutsumikoshi, S.; Yamauchi, K. Power Assist Bicycle. U.S. Patent 6,152,251, 28 November 2000.

23. Cohen, S. Social relationships and health. Am. Psychol. 2004, 59, 676. [CrossRef] [PubMed] 
24. Glei, D.A.; Landau, D.A.; Goldman, N.; Chuang, Y.L.; Rodriguez, G.; Weinstein, M. Participating in social activities helps preserve cognitive function: An analysis of a longitudinal, population-based study of the elderly. Int. J. Epidemiol. 2005, 34, 864-871. [CrossRef] [PubMed]

25. Fratiglioni, L.; Paillard-Borg, S.; Winblad, B. An active and socially integrated lifestyle in late life might protect against dementia. Lancet Neurol. 2004, 3, 343-353. [CrossRef]

26. Zunzunegui, M.V.; Alvarado, B.E.; Del Ser, T.; Otero, A. Social networks, social integration, and social engagement determine cognitive decline in community-dwelling Spanish older adults. J. Gerontol. Ser. B Psychol. Sci. Soc. Sci. 2003, 58, S93-S100. [CrossRef]

27. Kobayashi, H.; Katsura, S.; Ohnishi, K. An analysis of parameter variations of disturbance observer for motion control. IEEE Trans. Ind. Electron. 2007, 54, 3413-3421. [CrossRef]

28. Chen, M.; Shi, P.; Lim, C.C. Adaptive neural fault-tolerant control of a 3-DOF model helicopter system. IEEE Trans. Syst. Man Cybern. Syst. 2016, 46, 260-270. [CrossRef]

29. Salihbegovic, A.; Sokic, E.; Osmic, N.; Hebibovic, M. High performance disturbance observer based control of the nonlinear 2DOF helicopter system. In Proceedings of the International Symposium on Information, Communication and Automation Technologies (ICAT), Sarajevo, Bosnia and Herzegovina, 30 October-1 November 2013; pp. 1-7.

30. Oh, S.; Hata, N.; Hori, Y. Integrated motion control of a wheelchair in the longitudinal, lateral, and pitch directions. IEEE Trans. Ind. Electron. 2008, 55, 1855-1862.

31. RT.works. Available online: https://www.rtworks.co.jp/eng/index.html (accessed on 26 December 2020).

32. Tesio, L.; Rota, V.; Perucca, L. The 3D trajectory of the body centre of mass during adult human walking: Evidence for a speed-curvature power law. J. Biomech. 2011, 44, 732-740. [CrossRef]

33. Schouten, T.; Bolderdijk, J.; Steg, L. Framing car fuel efficiency: Linearity heuristic for fuel consumption and fuel-efficiency ratings. Energy Effic. 2014, 7, 891-901. [CrossRef]

34. Lee, H.S.; Tomizuka, M. Robust motion controller design for high-accuracy positioning systems. IEEE Trans. Ind. Electron. 1996, $43,48-55$.

35. Castell, M.V.; Sánchez, M.; Julián, R.; Queipo, R.; Martín, S.; Otero, Á. Frailty prevalence and slow walking speed in persons age 65 and older: Implications for primary care. BMC Fam. Pract. 2013, 14, 86. [CrossRef] [PubMed]

36. Hof, A.L. Scaling gait data to body size. Gait Posture 1996, 3, 222-223. [CrossRef]

37. Wobbrock, J.O.; Findlater, L.; Gergle, D.; Higgins, J.J. The aligned rank transform for nonparametric factorial analyses using only anova procedures. In Proceedings of the SIGCHI Conference on Human Factors in Computing Systems, Vancouver, BC, Canada, 7-12 May 2011; pp. 143-146. 